\title{
Human-mediated dispersal of cats in the Neolithic Central Europe
}

\author{
Mateusz Baca ${ }^{1}$ - Danijela Popović ${ }^{1}$ Hanna Panagiotopoulou ${ }^{2} \cdot$ Adrian Marciszak $^{3} \cdot$ Magdalena Krajcarz $^{4}$. \\ Maciej T. Krajcarz ${ }^{5}$. Daniel Makowiecki ${ }^{4}$ Piotr Węgleński ${ }^{1}$ - Adam Nadachowski ${ }^{6}$
}

Received: 30 January 2018 / Revised: 6 March 2018 / Accepted: 6 March 2018 / Published online: 28 March 2018

(c) The Genetics Society 2018

\begin{abstract}
Archeological and genetic evidence suggest that all domestic cats derived from the Near Eastern wildcat (Felis silvestris lybica) and were first domesticated in the Near East around 10,000 years ago. The spread of the domesticated form in Europe occurred much later, primarily mediated by Greek and Phoenician traders and afterward by Romans who introduced cats to Western and Central Europe around 2000 years ago. We investigated mtDNA of Holocene Felis remains and provide evidence of an unexpectedly early presence of cats bearing the Near Eastern wildcat mtDNA haplotypes in Central Europe, being ahead of Roman period by over 2000 years. The appearance of the Near Eastern wildcats in Central Europe coincides with the peak of Neolithic settlement density, moreover most of those cats belonged to the same mtDNA lineages as those domesticated in the Near East. Thus, although we cannot fully exclude that the Near Eastern wildcats appeared in Central Europe as a result of introgression with European wildcat, our findings support the hypothesis that the Near Eastern wildcats spread across Europe together with the first farmers, perhaps as commensal animals. We also found that cats dated to the Neolithic period belonged to different mtDNA lineages than those brought to Central Europe in Roman times, this supports the hypothesis that the gene pool of contemporary European domestic cats might have been established from two different source populations that contributed in different periods.
\end{abstract}

\section{Introduction}

These authors contributed equally: Mateusz Baca and Danijela Popović.

Electronic supplementary material The online version of this article (https://doi.org/10.1038/s41437-018-0071-4) contains supplementary material, which is available to authorized users.

\section{Mateusz Baca}

bacamat@gmail.com

1 Laboratory of Paleogenetics and Conservation Genetics, Centre of New Technologies, University of Warsaw, Banacha 2c, 02-079 Warsaw, Poland

2 Museum and Institute of Zoology, Polish Academy of Sciences, Wilcza 64, 00-679 Warsaw, Poland

3 Department of Paleozoology, Institute of Environmental Biology, University of Wroclaw, Sienkiewicza 21, 50-335 Wrocław, Poland

4 Institute of Archaeology, Faculty of History, Nicolaus Copernicus University, Szosa Bydgoska 44/48, 87-100 Torun, Poland

5 Institute of Geological Sciences, Polish Academy of Sciences, Research Centre in Warsaw, Twarda 51/55, 00-818 Warsaw, Poland

6 Institute of Systematics and Evolution of Animals, Polish Academy of Sciences, Sławkowska 17, 31-016 Krakow, Poland
Latest research on the wildcat Felis silvestris phylogeny resulted in distinction of five subspecies rank groups, corresponding to their geographical distribution (Driscoll et al. 2007, 2009): European wildcat, Southern African wildcat, Central Asian wildcat, Near Eastern wildcat and Chinese desert cat. Today, the European range of $F$. silvestris includes two subspecies. The European wildcat $(F$. s. silvestris) represents the only native form in most of the region. This animal was formerly widely distributed in Europe, except Fennoscandia (Yamaguchi et al. 2015), however, has become extinct in many areas mainly due to hunting and habitat loses. The second subspecies, domestic cat $(F$. s. catus), is of anthropogenic origin. The history of cat domestication was reconstructed with use of preserved written sources, art objects and archeozoological material and significantly supported during last years with genetic studies. According to genetic data, domestic cats do not descend from European wildcat, although European wildcat and domestic cat may share territory and crossbreed as interfertile taxa. The common ancestor to all modern domestic cats was the Near Eastern wildcat, F. s. lybica (Driscoll et al. 2007), domesticated in the Near East during 
Neolithic (Vigne et al. 2004; Driscoll et al. 2009; Faure and Kitchener 2009; Ottoni et al. 2017). The descendants of the domesticated Near Eastern wildcats were later spread across the world along with civilization expansion. Recently, analysis of mitochondrial DNA (mtDNA) from >200 Felis remains revealed that domestic cats originate from at least two different local populations of the Near Eastern wildcats, one from the Near East and the second from Egypt (Ottoni et al. 2017).

According to the current knowledge, the domestic cat did not occur in Central Europe prior to Roman Period (Benecke 1994; Clutton-Brock 1999; Driscoll et al. 2009; Faure and Kitchener 2009; Krajcarz et al. 2016), however, the chronology of the Near Eastern wildcat introduction to different regions of Europe is still weakly understood. The archeozoological and paleontological records are poor and direct chronometric data and ancient DNA analyses of fossil cats are still rare.

The preliminary study about the history of domestic cats in Poland (Krajcarz et al. 2016) revealed no presence of domesticated forms in archeological contexts before first century AD. Since that, study was focused on archeozoological material and did not include cat remains from non-human-related sites, there was a risk of overlooking the natural or civilization-related expansions of cats from the Near East. Here, we extended the prior survey to fossil Holocene cat's remains recovered from outside the archeological contexts.

\section{Materials and methods}

We analyzed bone fragments of 36 individuals from 19 sites in Poland (Supplementary Table S1) that were provisionally classified as Felis sp. or F. silvestris. This include six specimens excavated from archeological contexts for which partial mtDNA ND5 sequence was already published (Krajcarz et al. 2016). Sample handling and DNA extraction were performed in a laboratory dedicated to ancient DNA analyses in the Laboratory of Paleogenetics and Conservation Genetics, Centre of New Technologies at the University of Warsaw. Strict contamination precautions were undertaken during all steps of the experimental procedure. Prior to DNA extraction, each sample was washed with bleach solution ( $6 \% \mathrm{w} / \mathrm{v}$ sodium hypochlorite), rinsed with double distilled water, UV-irradiated $(245 \mathrm{~nm})$ for $20 \mathrm{~min}$ on each side and pulverized in cryogenic mill (SPEX CentriPrep, Stanmore, UK). DNA extraction was performed using modified silica column-based method optimized to retrieve short DNA fragments (Dabney et al. 2013). Samples were processed in batches of 16 with a negative control included in each batch. First, we screened all samples for DNA preservation by amplification of a short fragment of mitochondrial ND5 gene. Thirty-three samples yielded DNA sequence that allow initial species assignation (Supplementary Table 1). To obtain longer fragment of the mtDNA sequences, we used a targeted enrichment approach. For the hybridization experiment, we chose 20 samples, 12 that yielded $F$. s. lybica/catus and 8 that yielded F.s. silvestris haplotypes during initial screening. Those samples were either already radiocarbon dated or there was enough bone left to perform dating. DNA extracts were converted into double-indexed sequencing libraries following modified protocol of Meyer and Kircher (2010). To minimize sample cross-talk during sequencing, beside double indexing, we used adapters containing 7-bp long barcodes (Rohland et al. 2014). We targeted a 6-kb fragment of mtDNA genome spanning position from 11,487 to 925. Hybridization bait was produced from the DNA of a contemporary domestic cat. DNA from swab was extracted with DNeasy Blood \& Tissue Kit (Qiagen), and then the desired mtDNA fragment was amplified with three primer pairs. PCR products were sonicated to the length of around 200 bp with Covaris S220 and converted into bait following the protocol of Maricic et al. (2010). Hybridization was carried on pools of up to five libraries. We performed two rounds of hybridization for $21 \mathrm{~h}$ each following the protocol proposed by Horn (2012). Libraries were amplified for 19 cycles after the first and for 17 cycles after the second round. Enriched libraries were quantified with quantitative PCR (Illumina Library Quantification kit, KAPA), pooled in equimolar ratios and sequenced with other libraries on NextSeq or on MiSeq platform (Illumina) in the $2 \times 75$ bp or $2 \times 150$ modes, respectively. Libraries produced from extraction-negative controls were pooled, hybridized and sequenced as other libraries.

Sequencing reads were demultiplexed using Bcl2fastq, reads containing appropriate barcode were filtered with Sabre script, and then AdapterRemoval v. 2 (Lindgreen 2012) was used to collapse overlapping reads. Reads were mapped to cat reference mtDNA sequence using Bwa ( $\mathrm{Li}$ and Durbin 2010), only reads with mapping quality over 30 and longer than 30 bp were retained. Duplicates were removed; variants and consensus sequences were called using Samtools and Bcftools (Li et al. 2009). We called only positions with minimum $2 \times$ coverage. Each bam alignment was inspected manually in Tablet (Milne et al. 2013). Endogenous ancient DNA molecules typically exhibit excess of deaminated cytosines toward the ends of molecules; we used MapDamage v.2 (Jónsson et al. 2013) to check whether this pattern was present in the analyzed samples.

\section{Phylogenetic analyses}

To reconstruct the phylogenetic position of the analyzed cat remains, we used large dataset of sequences of 


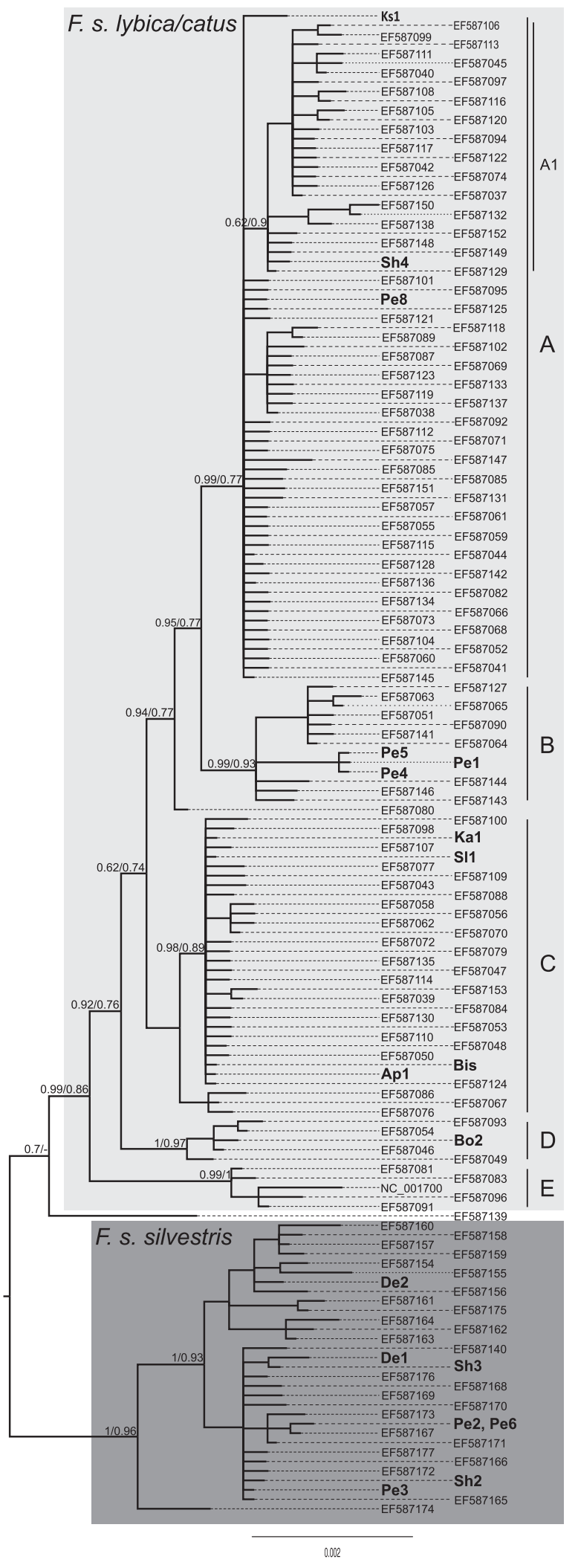

contemporary wildcats and domestic cats published by Driscoll et al. (2007). The final dataset consisted of 160 distinct haplotypes encompassing the 2604-bp long
Fig. 1 Phylogeny of Holocene and recent cats. Bayesian phylogeny based on $160 \mathrm{mtDNA}$ haplotypes of Holocene and recent cats. Haplotypes of studied samples are given in bold. Numbers at nodes indicate posterior probability and $\mathrm{SH}$ support values obtained with Bayesian and maximum likelihood approaches, respectively. The tree was rooted with sequence of Felis margarita (data not shown)

fragment between positions 12,642 and 15,245 of cat's mtDNA. Phylogenies were reconstructed with Bayesian and maximum likelihood methods using MrBayes 3.2.6 (Ronquist et al. 2012) and PhyML 3.1 (Guindon et al. 2010). Best partitioning scheme and substitution model for Bayesian analysis was found with PartitionFinder 2.1.1 (Lanfear et al. 2016) (Supplementary Table 2 and 3). The analysis consisted of two independent runs with four chains each, and was run for 10,000,000 generations with parameters sampled every 1000 generation. Stationarity and convergence were assessed in Tracer v. $1.6($ ESS $>200)$ (Rambaut and Drummond 2007). We also confirmed the average standard deviation of split frequencies to be below 0.01. In maximum likelihood analysis, the HKY $+\mathrm{G}$ substitution model was used as indicated by jModeltest 2 (Darriba et al. 2012). The best tree was chosen out of those obtained with nearest neighbor interchange and subtree pruning and regrafting tree rearrangement algorithms, approximate likelihood-ratio test with Shimodaira-Hasegawa ([SH]-aLRT) procedure was applied to assess branch support.

\section{Radiocarbon dating}

Radiocarbon dating of selected samples was performed in Poznan Radiocarbon Laboratory using accelerator mass spectrometry method. Obtained ${ }^{14} \mathrm{C}$ dates were calibrated in OxCal v. 4.2.4 (Bronk Ramsey 2009) using IntCal13 calibration curve (Reimer et al. 2013).

\section{Results}

Out of 20 samples used in hybridization capture experiment, 18 produced targeted mtDNA fragment with minimum of $70 \%$ sites covered at least two times and those samples were used in phylogenetic reconstruction (Supplementary Table 4). In case of sample Lo1, the recovered fragment of mtDNA was too short to be used in phylogenetic reconstruction but confirmed subspecies assignation. DNA molecules of majority of the samples exhibit damage pattern typical for ancient DNA, only in case of two youngest samples Bis and Ap1 the pattern was questionable (Supplementary Fig. 1). This is, however, expected, as the amount of damage is the function of time after deposition. Careful examination of bam alignments revealed no signs of 
contamination. There was also no reads mapping to cat's mtDNA genome in extraction negative controls.

Reconstructed phylogeny correspond to this obtained earlier by Driscoll et al. (2007) with clearly separated lineages of European wildcats and Near Eastern wildcats/ domestic cats with five sublinages (A-E) distinguished within the latter (Fig. 1). Within sublineage A, a branch recently marked as A1 by Ottoni et al. (2017) was observed with moderate support values. Phylogenetic analyses confirmed the initial subspecies assignation and 11 samples were classified as $F$. s. lybica/catus and seven as $F$. s. silvestris (Fig. 1). Out of 11 specimens with F. s. lybica/catus mtDNA haplotypes, two specimens yielded modern, two Late Medieval, one Early Medieval and one Roman ages according to radiocarbon dating, while the five other yielded surprisingly early ages of Middle to Late Neolithic, ranging between 5300 and 4200 years cal BP (Fig. 2; Supplementary Table 5). The reliability of dating was confirmed by measurements of the $\mathrm{C} / \mathrm{N}$ ratio in collagen, which was in accepted range (2.9-3.6) (DeNiro 1985). Only in case of one Neolithic sample, the collagen yield was too low to confirm quality of the dated material (Supplementary Table 5). Those five samples come from three paleontological sites, Shelter in Krucza Skala (Ks1), Perspektywiczna Cave (Pe1, Pe4 and Pe5) and Shelter in Smoleń III (Sh4) and were not associated with cultural remains. Sample Ks1 belonged to sublineage A, Sh4 to A1, whereas samples Pe1, $\mathrm{Pe} 4$ and Pe5 to sublineage B (Supplementary Table 6). Samples Pe1, Pe4 and Pe5 yielded similar radiocarbon dates and identical mtDNA haplotypes, although bones come from different, non-contiguous layers and distant parts of the site, we cannot exclude possibility that they belong to the single individual. F. s. lybica/catus specimens dated to the Roman period until modern times comes both from anthropogenic (Ka1, S11, Bis and Bo2) and paleontological (Ap1 and Pe8) contexts (Supplementary Table 1). Most of them belonged to mtDNA sublineage $\mathrm{C}$ (Ka1, S11, Bis and Ap1), whereas Bo2 belonged to sublineage D and Pe8 to A.

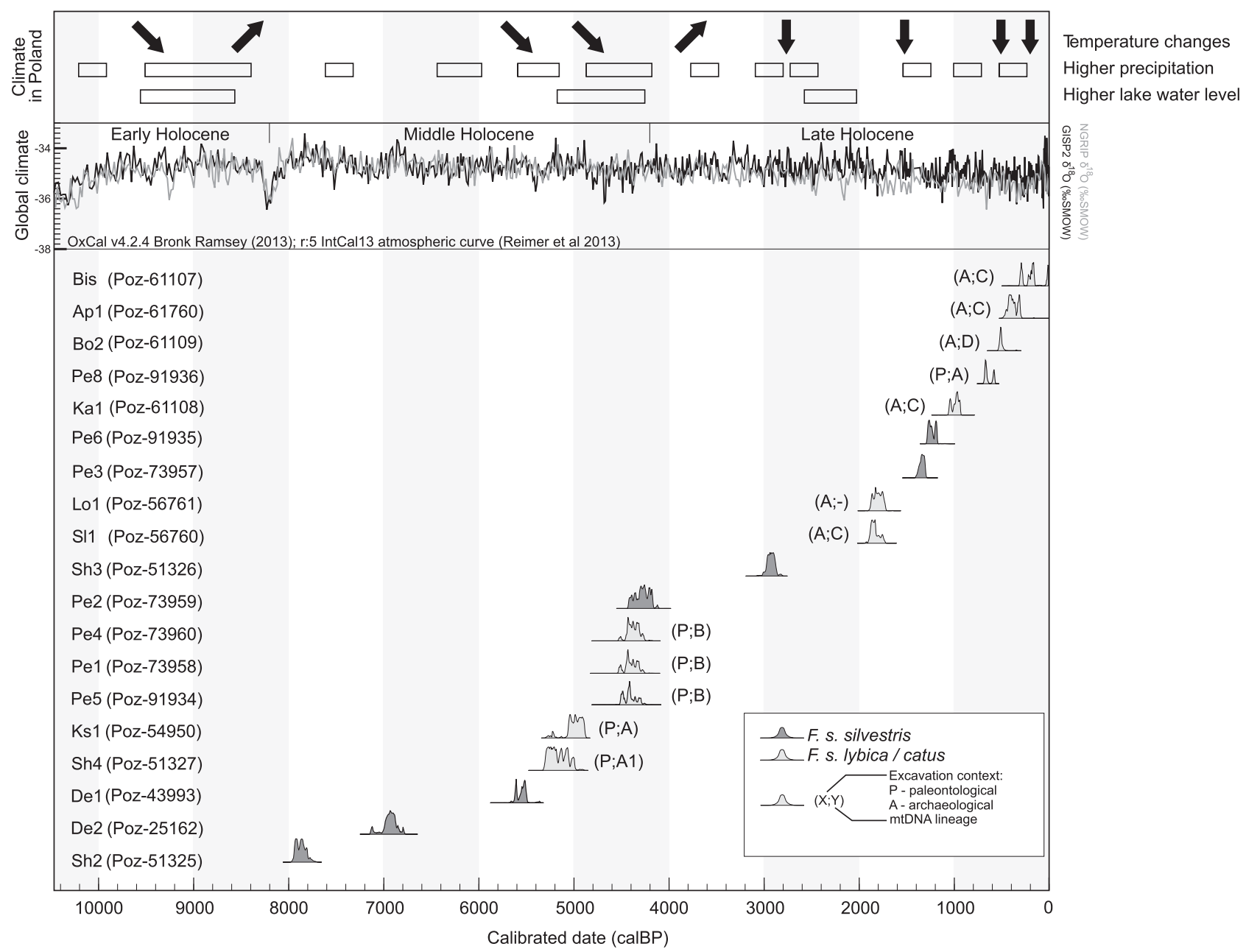

Fig. 2 Calibrated radiocarbon ages of the Holocene cat remains. Calibration and $\delta^{18} \mathrm{O}$ curves are given according to Bronk Ramsey (2009). The climatic proxies set in the same scale are given after
Starkel et al. (2006, 2013). Information about excavation context and mtDNA lineage of F.s. lybica/catus specimens are given next to age probability distribution 


\section{Discussion}

Available archeological data suggest that domestic cats made their way to Greece and Rome with Phoenician traders not earlier than 3400 and 2500 years BP, respectively (Faure and Kitchener 2009). Their subsequent spread throughout Europe was mediated by growing Roman Empire and took place around 2000 years BP, thus the appearance of $F$. s. lybicalcatus haplotypes in Poland already in Neolithic period was highly unexpected. Due to the lack of anthropogenic context, the studied Felis remains dated to Neolithic period, cannot be easily associated with humans, and other scenarios that led to the ancient occurrence of those haplotypes in Central Europe need to be considered as well. First, their presence could have resulted from natural expansion of $F$. s. lybica from the Near East during the period of favorable climatic conditions. Second, it could have resulted from ancient hybridization between European and the Near Eastern wildcats, and subsequent spread of the introgressed individuals into Central Europe. Finally, the Near Eastern wildcat specimens might have followed humans as synanthropic commensals during the expansion of Neolithic cultures.

The first scenario seems the least likely one. Near Eastern wildcats inhabit mostly hot and dry climatic zones of Northern Africa and Arabian Peninsula with steppe environments including savannas and shrub grasslands (Yamaguchi et al. 2015). Paleoclimate data suggest that the period when the Near Eastern wildcat's haplotype appeared in Poland was characterized by relatively cool and moist climate with high rate of precipitation and elevated water level (Starkel et al. 2006, 2013) (Fig. 2). This, together with cooccurrence of native European wildcat that mostly inhabits forests, makes the natural expansion of $F$. s. lybica into territory of modern Poland implausible.

The second scenario that assumes ancient hybridization of European and the Near Eastern wildcats is more credible. Nowadays, hybridization between European wildcats and feral domestic cats is common (Randi et al. 2001; Oliveira et al. 2008; Hertwig et al. 2009; Mattucci et al. 2013). Moreover, Driscoll et al. (2007) found 28 individuals with domestic cat mtDNA among 108 individuals with purely European wildcat nuclear DNA. Such mito-nuclear discordance was interpreted as a result of hybridization event between wildcat subspecies that might have taken place shortly after the domestic cats were brought into the range of European wildcats. Recently, F. s. lybica haplotypes were found also in pre-Neolithic Romania, those individuals belonged exclusively to mitochondrial lineage A1 (Ottoni et al. 2017). This finding led authors to conclusion that since the beginning of Holocene, the natural range of Near Eastern wildcats was wider and included also Southeastern Europe, which also became a historical hybrid zone for
European and Near Eastern wildcats. In consequence, the mitochondrial haplotypes of the $F$. s. lybica/catus might have spread in the $F$. s. silvestris populations in Europe. Similar mito-nuclear discordances were observed in other mammalian taxa and interpreted as a result of hybridization after temporary contact between their populations in the past (Alves et al. 2008; Toews and Brelsford 2012).

Interestingly, the dating of the oldest remains with $F . s$. lybica/catus haplotypes coincides with the appearance of the early farmers in Poland. The earliest Neolithic settlements of Linear Band Pottery culture in Poland appeared around 7500 years cal BP (Czekaj-Zastawny 2017). The peak of Neolithic settlement density falls between 5500 and 4500 years cal BP in Kuyavia and between 5000 and 4000 in Lesser Poland (Timpson et al. 2014). This leads to the third scenario that hypothesizes a spread of the Near Eastern wildcat throughout Europe as a commensal form that followed human groups during the dispersal of Neolithic cultures. The similar way of spread alongside early farmers was recently well documented for early-domesticated pigs (Larson et al. 2007; Ottoni et al. 2013). Processes of wildcat and wild boar domestication have followed the similar, that is, commensal pathway (Larson and Fuller 2014). In its early stages, during Early Holocene, cats and boars had been attracted to human settlements by food wastes and pests without any deliberate humans activities (Driscoll et al. 2009). Pigs were, however, recognized as a valuable resource and domesticated much earlier than cats, which remained mostly commensal species for next several thousands of years (Larson and Fuller 2014). The expansion of wildcats to Europe as commensal animals together with early Neolithic groups might have resulted in the observed pattern with Near Eastern wildcat remains found in paleontological contexts not related with humans. Phylogenetic position of $F$. s. lybica/catus individuals from Neolithic Poland supports this scenario, although the presence of lineage A1 may have resulted from introgression of European wildcats with natural population of Near Eastern wildcats in Southeast Europe. However, the presence of lineages A and B cannot be easily explained this way. Lineage A was the main lineage, which was domesticated in the Near East and which is the most frequent lineage in recent domestic cats. Individual belonging to this lineage was reported in Early Neolithic Bulgaria around 6400 years $\mathrm{BP}$, what was also interpreted as a result of human-mediated dispersal (Ottoni et al. 2017). Lineage B, the second domesticated lineage, was found so far only in Southeast Anatolia, Jordan and Iran. Given that in the dataset by Ottoni et al. (2017), there is not a single instance of European wildcat in Anatolia, it is unlikely that presence of those lineages in Central Europe may have resulted from introgression between Felis subspecies. This suggests rather a scenario where the Near Eastern wildcats spread together 
with early farmers from Anatolia first to Southeast Europe where they crossbreed with local population and acquired lineage A1 and then further northwest to Central Europe. There is also, however, a range of possible intermediate scenarios that cannot be ruled out, such as hybridization between European and Near Eastern wildcats after arrival of early farmers (i.e., haplogroups $\mathrm{A}$ and possibly B) to Southeast Europe.

Interesting is the apparent discontinuity between Neolithic and younger samples. Although based on a limited sample size, it suggests that the cats from Neolithic period stem from a different source population than domestic cats brought to Central Europe by Romans and that the gene pool of contemporary European domestic cats might have been established from the two different source populations that contributed in the two different periods. This is in line with the findings by Ottoni et al. (2017) who showed that cats introduced to Europe during Classical times belonged mostly to lineage $\mathrm{C}$ domesticated in Egypt.

Investigation of mtDNA from Holocene Felis remains revealed $F$. s. lybica/catus haplotypes present in Central Europe already in Neolithic period. The available data do not allow for certain discrimination between alternatives explaining their presence, however, supports dispersal mediated by humans. This transforms current knowledge and poses new questions about the history of domestic cats in Europe. As there is no evidence for domestic cats in archeological record prior to Roman Period, how and to what extent cats that spread in Europe during Neolithic participated in the gene pool of contemporary cats? Further investigation of Holocene and recent cats with a panel of nuclear markers would enable tracing the ancestry of contemporary domestic cats.

\section{Data archiving}

Nucleotide sequences reported in this study were deposited in GenBank under accession no. MG813950-MG813967. Bam alignments generated in the study are stored in ENA repository under project accession no. PRJEB25368

\begin{abstract}
Acknowledgements We acknowledge Anna Baca for her help in editing and proofreading of the manuscript. Cat remains from Perspektywiczna Cave and Shelter in Smoleń III were collected during field works supported by the National Science Centre, Poland, grant numbers 2011/01/N/HS3/01299 and 2014/15/D/HS3/01302, and radiocarbon dating was supported by the National Science Centre, Poland, grant number 2014/13/D/HS3/03842. MB was supported by the National Science Centre, Poland, grant number 2015/19/D/NZ8/ 03878 .
\end{abstract}

Author contributions $\mathrm{MB}$ and $\mathrm{MK}$ conceived and coordinated the study; MK, MTK, AM, AN and DM provided samples and radiocarbon dating; DP, HP, PW and MB participated in laboratory work; MB and DP carried out the phylogenetic analyses; MB, MK and MTK wrote the manuscript with significant input from all the authors. All authors gave final approval for publication.

\section{Compliance with ethical standards}

Conflict of interest The authors declare that they have no conflict of interest.

\section{References}

Alves PC, Melo-Ferreira J, Freitas H, Boursot P (2008) The ubiquitous mountain hare mitochondria: multiple introgressive hybridization in hares, genus Lepus. Philos Trans R Soc Lond B Biol Sci 363:2831-2839

Benecke N (1994) Der Mensch und seine Haustiere. Die Geschichte einer jahrtausendealten Beziehung. Konrad Theiss-Verlag, Stuttgart

Bronk Ramsey C (2009) Bayesian analysis of radiocarbon dates. Radiocarbon 51:337-360

Clutton-Brock J (1999) A natural history of domesticated mammal. Natural History Museum, London

Czekaj-Zastawny A (2017) The first farmers from the south - Linear Pottery culture. In: Urbańczyk P (ed) The past societies. Polish lands from the first evidence of human presence to the Early Middle Ages 2: 5500-2000 BC. Institute of Archaeology and Ethnology, Polish Academy of Science, Warsaw

Dabney J, Knapp M, Glocke I, Gansauge M-T, Weihmann A, Nickel $\mathrm{B}$ et al. (2013) Complete mitochondrial genome sequence of a Middle Pleistocene cave bear reconstructed from ultrashort DNA fragments. Proc Natl Acad Sci USA 110:15758-15763

Darriba D, Taboada GL, Doallo R, Posada D (2012) jModelTest 2: more models, new heuristics and parallel computing. Nat Methods 9:772-772

DeNiro M (1985) Postmortem preservation and alteration of in vivo bone collagen isotope ratios in relation to palaeodietary reconstruction. Nature 317:806-809

Driscoll CA, Macdonald DW, O'Brien SJ (2009) From wild animals to domestic pets, an evolutionary view of domestication. Proc Natl Acad Sci USA 106(Suppl):9971-9978

Driscoll CA, Menotti-Raymond M, Roca AL, Hupe K, Johnson WE, Geffen E et al. (2007) The Near Eastern origin of cat domestication. Science 317:519-523

Faure E, Kitchener AC (2009) An archaeological and historical review of the relationships between felids and people. Anthrozoos 22:221-238

Guindon S, Dufayard J-F, Lefort V, Anisimova M, Hordijk W, Gascuel O (2010) New algorithms and methods to estimate maximum-likelihood phylogenies: assessing the performance of PhyML 3.0. Syst Biol 59:307-321

Hertwig ST, Schweizer M, Stepanow S, Jungnickel A, Bohle UR, Fischer MS (2009) Regionally high rates of hybridization and introgression in German wildcat populations (Felis silvestris, Carnivora, Felidae). J Zool Syst Evol Res 47:283-297

Horn S (2012) Target enrichment via DNA hybridization capture. In: Shapiro B, Hofreiter M (eds) Ancient DNA. Methods and protocols, methods in molecular biology (Clifton, N.J.). Humana Press, Totowa, NJ, p 189-195. Vol 840

Jónsson H, Ginolhac A, Schubert M, Johnson PLF, Orlando L (2013) MapDamage2.0: fast approximate Bayesian estimates of ancient DNA damage parameters. Bioinformatics 29:1682-1684

Krajcarz M, Makowiecki D, Krajcarz MT, Masłowska A, Baca M, Panagiotopoulou H et al. (2016) On the trail of the oldest domestic cat in Poland. An insight from morphometry, ancient DNA and radiocarbon dating. Int J Osteoarchaeol 26:912-919 
Lanfear R, Frandsen PB, Wright AM, Senfeld T, Calcott B (2016) PartitionFinder 2: new methods for selecting partitioned models of evolution for molecular and morphological phylogenetic analyses. Mol Biol Evol 34:msw260

Larson G, Cucchi T, Fujita M, Matisoo-smith E, Robins J, Anderson A et al. (2007) Phylogeny and ancient DNA of Sus provides insights into neolithic expansion in Island Southeast Asia and Oceania. Proc Natl Acad Sci USA 104:4834-4839

Larson G, Fuller DQ (2014) The evolution of animal domestication. Annu Rev Ecol Evol Syst 45:115-136

Li H, Durbin R (2010) Fast and accurate long-read alignment with Burrows-Wheeler transform. Bioinformatics 26:589-595

Li H, Handsaker B, Wysoker A, Fennell T, Ruan J, Homer N et al. (2009) The sequence alignment/map format and SAMtools. Bioinformatics 25:2078-2079

Lindgreen S (2012) AdapterRemoval: easy cleaning of next-generation sequencing reads. BMC Res Notes 5:337

Maricic T, Whitten M, Pääbo S (2010) Multiplexed DNA sequence capture of mitochondrial genomes using PCR products. PLoS ONE 5:9-13

Mattucci F, Oliveira R, Bizzarri L, Vercillo F, Anile S, Ragni B et al. (2013) Genetic structure of wildcat (Felis silvestris) populations in Italy. Ecol Evol 3:2443-2458

Meyer M, Kircher M (2010) Illumina sequencing library preparation for highly multiplexed target capture and sequencing. Cold Spring Harb Protoc 5:t5448

Milne I, Stephen G, Bayer M, Cock PJA, Pritchard L, Cardle L et al. (2013) Using Tablet for visual exploration of second-generation sequencing data. Brief Bioinform 14:193-202

Oliveira R, Godinho R, Randi E, Ferrand N, Alves PC (2008) Molecular analysis of hybridisation between wild and domestic cats (Felis silvestris) in Portugal: implications for conservation. Conserv Genet 9:1-11

Ottoni C, Girdland Flink L, Evin A, Georg C, De Cupere B, Van Neer W (2013) Pig domestication and human-mediated dispersal in western Eurasia revealed through ancient DNA and geometric morphometrics Mol Biol Evol 30:824-832
Ottoni C, Van Neer W, De Cupere B, Daligault J, Guimaraes S, Peters $\mathrm{J}$ et al. (2017) The palaeogenetics of cat dispersal in the ancient world. Nat Ecol Evol 1:139

Rambaut A, Drummond AJ (2007). Tracer v. 1.5. MCMC trace analysis tool. Available at: http://tree.bio.ed.ac.uk/software/tracer.

Randi E, Pierpaoli M, Beaumont M, Ragni B, Sforzi A (2001) Genetic identification of wild and domestic cats (Felis silvestris) and their hybrids using Bayesian clustering methods. Mol Biol Evol 18:1679-1693

Reimer PJ, Bard E, Bayliss A, Beck JW, Blackwell PG, Bronk C et al. (2013) Intcal13 and marine13 radiocarbon age calibration curves 0 - 50,000 years cal bp. Radiocarbon 55:1869-1887

Rohland N, Harney E, Mallick S, Nordenfelt S, Reich D (2014) Partial uracil-DNA-glycosylase treatment for screening of ancient DNA. Philos Trans R Soc B Biol Sci 370:20130624-20130624

Ronquist F, Teslenko M, van der Mark P, Ayres DL, Darling A, Höhna $S$ et al. (2012) MrBayes 3.2: efficient Bayesian phylogenetic inference and model choice across a large model space. Syst Biol 61:539-542

Starkel L, Michczyńska DJ, Krąpiec M, Margielewski W, Nalepka D, Pazdur A (2013) Progress in the Holocene chronoclimatostratigraphy of Polish territory. Geochronometria 40:1-21

Starkel L, Soja R, Michczyńska DJ (2006) Past hydrological events reflected in Holocene history of Polish rivers. Catena 66:24-33

Timpson A, Colledge S, Crema E, Edinborough K, Kerig T, Manning $\mathrm{K}$ et al. (2014) Reconstructing regional population fluctuations in the European Neolithic using radiocarbon dates: a new case-study using an improved method. J Archaeol Sci 52:549-557

Toews DPL, Brelsford A (2012) The biogeography of mitochondrial and nuclear discordance in animals. Mol Ecol 21:3907-3930

Vigne J-D, Guilaine J, Debue K, Haye L, Gerard P (2004) Early taming of the cat in Cyprus. Science 304:259

Yamaguchi N, Kitchener AC, Driscoll CA, Nussberger B (2015). Felis silvestris. The IUCN Red List of Threatened Species. e. T60354712A50652361. http://dx.doi.org/10.2305/IUCN.UK.20152.RLTS.T60354712A50652361.en. 